Abb. 7 faßt diesen Verlauf nochmals an einer der ganzen Platte entsprechenden Gesamtheit von Ellipsen und Kreisen zusammen.

Es wird besonders an dem letzten allgemeinsten Beispiel, an dem jeder Ast stetig von Maximis zu Minimis führt, deutlich, wie sekundär diese Kennzeichnung durch Extrema überhaupt ist. Das stetig Verbindende einer dieser Kurven des Glanzbereichs ist die Tatsache des Berührens des beugenden Spaltes mit einer der Flächen konstanter Lichtzeit, die Gleichheit der benachbarten Lichtwege.

Zieht man in Abb. 5 die Grenze, außerhalb deren die Glanzbereiche nur noch einen Ast zeigen, so ergibt sich die in $\mathrm{I}^{1}$, Abb. 4, gezeigte Kaustik. Die größere Helligkeit im inneren Feld rührt also daher, daß hier ein größerer Teil der beugenden Platte mitarbeitet.

5. Die Gewöhnung an Gitter führt, wie Gespräche mehrfach zeigten, auf die Versuchung, die statistische Platte als ein Gitter aufzufassen, dessen Konstante um einen Mittelwert schwankt. Das ist ebensowenig sachgemäß, wie wenn man die freien Flugzeiten von Gasmolekülen als Schwankungen um ihren Mittelwert auffassen und irgendwelche auf diese mittlere Flugzeit bezogenen Resonanzerscheinungen erwarten wollte. Der Vorgang an der statistischen Platte ist durchaus $\left(\mathrm{I}^{1}, 8\right)$ als Intensitätsüberlagerung der
Beugungsbilder von Kreisrändern aufzufassen. die diese sonst lichtschwache Erscheinung intensiv zeigt und ihren Verlauf mit der Größe des beugenden Randes vorführt.

Andererseits ist es, sobald dies klar feststeht, instruktiv, zum Beugungsbild äquidistanter Kreisspalte, also eines Kreisgitters, weiterzugehen $\left(\mathrm{I}^{1}, 6\right)$. Bemerkenswerterweise erhält man die dafür bezeichnenden Erscheinungen auch, wenn nicht in zeitraubenden Einzeleinstellungen einzelne Kreise gezogen werden, sondern ebenso von einer einzigen Furche von gleichförmig ansteigendem Radius. Man hat hier den Vorteil, daß die Drehbank automatisch arbeitet, indem der Diamant mittels des Planschubes in einer Spirale konstanter Ganghöhe einwärts geschoben wird. Dies erfordert natürlich eine sorgfältige, in der Druckrichtung elastische Halterung des Diamanten, die es erlaubt, den für das Ritzen maßgebenden Druck willkürlich zu justieren. Hier war die Erfahrung von Hrn. Obermeister S pei del von entscheidender Bedeutung. Am besten befriedigten Ganghöhen um $11 \mu$; bei der engsten Ganghöhe, die sich einstellen ließ, $7 \mu$, beeinträchtigen augenscheinlich die unter dem Mikroskop sichtbaren Ganghöhenschwankungen um einige $\mu$ bereits die Reinheit der optischen Wirkung.

Hrn. Obermeister Speidel und Hrn. cand. phys. F. Sa u r danke ich für ihre Mitwirkung.

\title{
Zur Lichtbeugung an statistischen Ringplatten
}

\author{
Von Werner BraunbeK ${ }^{1}$ \\ (Z. Naturforschg. 4 a, 509-515 [1949]; eingegangen am 8. März 1949)
}

\begin{abstract}
Das Beugungsfeld der von Kossel angegebenen Platte mit konzentrischen Ringen statistisch verteilter Radien wird untersucht. Für den „Brennstrahl“ wird die axiale Intensitätsverteilung sowie der Abfall nach außen berechnet, wofür sich sehr einfache Näherungsausdrücke ergeben. Auch das Wiederauftreten des „Brennstrahls“ hinter einem Hindernis sowie sein Erlöschen hinter einer Lochblende werden diskutiert.
\end{abstract}

\section{Problem und Voraussetzungen}

$\mathrm{D}^{\mathrm{i}}$ ie Beugung durch ein System konzentrischer Kreisringe ist vor allem durch die So retsche Zonenplatte bekannt, bei welcher durch eine ganz bestimmte Folge der Kreisradien - diese verhalten sich wie die Wurzeln aus der Folge der ganzen Zahlen - erreicht wird, daß bei Beleuchtung

1 Universität Tübingen.

2 W. Kossel, Z. Naturforschg. 3a; 496 [1948]. von einer punktförmigen Lichtquelle auf der Achse aus im Beugungsfeld sich die ganze Intensität auf die engste Umgebung gewisser weniger Achspunkte konzentriert, während sich im übrigen Raum die Beiträge der verschiedenen Ringe weitgehendst wegheben. Aber auch andere Ringanordnungen liefern interessante Erscheinungen; insbesondere hat kürzlich $\mathrm{Kossel}^{2}$ eine Ringplatte mit konzentrischen Ringen angegeben, deren Radien statistisch verteilt sind, und deren 
Eigenschaften er auch in der hier unmittelbar voranstehenden Arbeit weiter bespricht.

Eine solche Platte ergibt, von einem Lichtpunkt auf der Achse beleuchtet, entlang der Achse einen sehr scharfen „Brennstrahl“, der aber kein richtiger Strahl ist, sondern eine kontinuierliche Folge von Beugungsmaximis nullter Ordnung, der daher hinter einem kleinen Hindernis wieder erneut auftritt, hinter einer Lochblende dagegen erlischt.

Den Intensitätsverlauf hinter einer solchen Platte bei achsenparalleler Beleuchtung (bei einer Punktquelle auf der Achse in endlicher Entfernung sind die Erscheinungen nicht grundsätzlich verschieden) wollen wir hier unter folgenden idealisierenden Voraussetzungen berechnen:

(1) Die einzelnen Ringe nehmen wir als unendlich schmal an, jedoch unter sich gleich breit, und darüber hinaus als gleichphasig kohärent leuchtende Kreislinien, so daß der sonst in dem Kirch$\mathrm{h}$ of f schen Integral auftretende Faktor $(1+\cos \psi)$ durch eine Konstante ersetzt werden darf. Dadurch wird die Rechnung wesentlich einfacher und übersichtlicher, während andererseits z. B. wegen des Einflusses der Furchenform auf den Richtungsfaktor auch die Mitnahme des Faktors $(1+\cos \psi)$ keine ganz zuverlässigen Ergebnisse zeitigen würde, und die spezielle Form dieses Faktors sowieso wenig Einfluß auf den prinzipiellen Intensitätsverlauf hat.

(2) Die Radien einer großen Zahl $N$ von Ringen sollen gleich wahrscheinlich, d. h. mit einer konstanten Verteilungsfunktion zwischen einer unteren Grenze $R_{1}$ und einer oberen Grenze $R_{2}$ verteilt sein. Es ist hierbei zweckmäßig, $R_{1}$ nicht gleich Null, sondern von derselben Größenordnung wie $R_{2}$, jedoch auch nicht $z u$ nahe an $R_{2}$ anzunehmen. Auch bei der experimentellen Untersuchung solcher Scheiben wird ja meist die zentrale Zone bis zu einem Radius $R_{1}$ abgedeckt.

Der statistischen Natur des Problems entsprechend, errechnen wir den Erwartungswert $\overline{S^{2}}$ der Lichtintensität $S^{2}$ an irgendeinem Raumpunkt. Die wirkliche Intensität, welche eine individuelle Platte an diesem Punkt liefert, wird sich davon möglicherweise um eine Größenordnung unterscheiden, die als Schwankung um den Erwartungswert relativ zu diesem mit $1 / \sqrt{N}$ geht, also bei großer Ringzahl vernachlässigbar ist.

Die in den Voraussetzungen (1) und (2) benutzte Art der Statistik entspricht natürlich nicht ganz den wirklichen Verhältnissen. Insbesondere sind ja sicher nicht nur die Ringradien, die sich vor allem auf die Phasen der. Teilwellen auswirken, statistisch verteilt, sondern es streuen auch die Amplituden der von den einzelnen Ringen ausgehenden Teilwellen, welche wir hier einfach ohne Streuung als konstant (pro Längenelement jedes Ringes) angesehen haben. Diese Vereinfachung hat jedoch sicher keinen nennenswerten Einfluß auf das Ergebnis, da wir uns z. B. zunächst alle Ringe ungefähr gleicher TeilwellenAmplitude herausgreifen könnten und für diese allein - die ja auch schon gleichwahrscheinlich zwischen $R_{1}$ und $R_{2}$ verteilt sind - bereits das ganze Beugungsfeld erhalten würden, so daß zum Schluß die Überlagerung der unter sich gleichen Beugungsfelder der verschiedenen herausgegriffenen Ringgruppen nichts Neues liefern könnte.

\section{Das Beugungsfeld des einzelnen}

$$
\mathrm{R} \text { ing } \mathrm{s}
$$

Mit der Annahme (1). wird in bekannter Weise die von einem einzelnen Ring herrührende komplexe Amplitude $\mathfrak{A}$ in irgendeinem Raumpunkt $\mathrm{P}$ (Abb. 1)

$$
\mathfrak{U} \sim R \int_{0}^{2 \pi} \frac{d \vartheta}{\varrho} \exp (-i k g),
$$

falls $k=2 \pi / \lambda$ die Wellenzahl der Strahlung und $\varrho$ der Abstand des beugenden Elements $Q$ vom Aufpunkt $P$ ist, und wir von vornherein uns auf Entfernungen vom beugenden Ring $\gg \lambda$ beschränken, so daß im Kirchhoffschen Integral das 1/@ gegenüber $k$ vernachlässigt werden darf.

Das Integral in (3) ist nur geschlossen auswertbar, falls die Entwicklung von $\varrho=\sqrt{s^{2}-2 R y \cos \vartheta}$ nach $y$ mit dem in $y$ linearen Glied abgebrochen werden darf, wenn also

ist.

$$
R y \ll s^{2}, \quad\left(s^{2}=x^{2}+y^{2}+R^{2}\right)
$$

Da die Beziehung $R y=\alpha s^{2}(\alpha \ll 1)$ einen Kreis in der $x-y$-Ebene darstellt, welcher die $y$ Achse in $y_{1} \ll R$ und $y_{2} \gg R$ schneidet, bedeutet die Bedingung (4) den Ausschluß eines Torus, welcher durch Rotation des eben erwähnten Kreises um die $x$-Achse entsteht und den beugenden Kreis einschließt. Der gesamte Raum außerhalb dieses Torus wird durch die folgende, bekannte Näherung beherrscht, welche mit der Entwicklung von $\varrho$ aus (3) liefert: 
$\mathfrak{A} \sim R \frac{\exp (-i k s)}{s}\left[J_{0}\left(\frac{k R y}{s}\right)+i \frac{R y}{s^{2}} J_{1}\left(\frac{k R y}{s}\right)\right]$,

mit den Bessel-Funktionen $J_{0}$ und $J_{1}$, bzw.

$$
\mathfrak{A} \sim R \frac{\exp (-i k s)}{s} J_{0}\left(\frac{k R y}{s}\right)
$$

da wir wegen (4) das 2. Glied wegstreichen können.

Mit (5) ist die komplexe Amplitude im ganzen Beugungsfeld ( $s$ ist Funktion von $x$ und $y$; das Beugungsfeld ist rotationssymmetrisch um die $x$ Achse) bekannt, und nun können wir die Felder der $N$ Ringe überlagern.

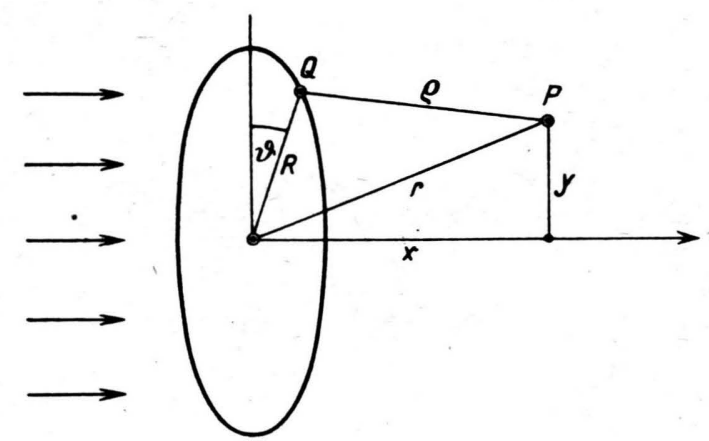

Abb.1. Zur Beugung am einzelnen Kreisring. Bezeichnungen: Ringachse $=x$-Achse; $y=$ Achsabstand des Aufpunktes $\mathrm{P} ; \quad R=$ Ringradius; $r^{2}=x^{2}+y^{2}$; $s^{2}=x^{2}+y^{2}+R^{2}=r^{2}+R^{2} ; \varrho^{2}=s^{2}-2 R y \cos \vartheta$.

3. Diestatistische Seitedes Problems

Wenn wir für einen festen Raumpunkt $(x, y)$ die komplexen Amplituden $\mathfrak{A}_{k}$ der $N$ Ringe addieren, könnten wir zunächst vermuten, der Erwartungswert $\overline{S^{2}}$ des Betragsquadrats der Summe sei einfach die Summe der Betragsquadrate der einzelnen $\mathfrak{A}_{k}$, da ja wegen der statistischen Verteilung der Ringradien $R_{k}$ die Phasen $k s_{k}$ ( $s$ hängt von $R$ ab!) ebenfalls statistisch verteilt sind, und Lichterregungen, welche mit statistisch verteilten Phasen zusammentreffen, einfach nach Intensitäten, also Betragsquadraten der komplexen Amplituden, $\mathrm{zu}$ addieren sind.

Nun liegen jedoch die Dinge hier nicht ganz so einfach. Einmal entspricht nämlich der gleichwahrscheinlichen Verteilung $\operatorname{der} R_{k}$ wegen der nicht linearen Abhängigkeit des $s$ von $R$ keine gleichwahrscheinliche Verteilung der Phasen $k s_{k}$ und zum andern sind auch die Beträge $\frac{R}{s} J_{0}$ der $\mathfrak{A}_{k}$ komplizierte und wegen der Bessel-Funktion im allgemeinen sogar rasch veränderliche Funktionen von $R$, so daß auch sie einer statistischen Verteilung unterliegen, welche aber mit derjenigen der Phasen gekoppelt ist, da beide von demselben Parameter $R$ abhängen.

Wir haben demnach folgende Frage zu beantworten: Welches ist der Erwartungswert $\bar{S}^{2}$ des Betragsquadrats einer Summe von $N$ komplexen Amplituden $\mathfrak{a}_{k} \exp \left(i \varphi_{k}\right)$, deren Betrag $a$ und deren Phase $\varphi$ je eine eindeutige Funktion desselben Parameters $R$ sind, wenn dieser Parameter $R$ statistisch zwischen den Grenzen $R_{1}$ und $R_{2}$ mit Gleichwahrscheinlichkeit für jedes Intervallelement verteilt ist?

Die Lösung dieser Frage, welche man durch Ausrechnen der Summe und Bildung des Erwartungswertes jedes einzelnen Summengliedes nach dem üblichen Verfahren erhält, wobei zwei Gruppen unter sich gleicher Summenglieder entstehen, die sich zusammenfassen lassen, ist:

$$
\begin{aligned}
& \overline{S^{2}}=\frac{N}{R_{2}-R_{1}} \int_{R_{1}}^{R_{2}} a^{2} d R+ \\
& +\frac{N(N-1)}{\left(R_{2}-R_{1}\right)^{2}}\left\{\left[\int_{R_{1}}^{R_{2}} a \cos \varphi d R\right]^{2}+\left[\int_{R_{1}}^{R_{2}} a \sin \varphi d R\right]^{2}\right\}
\end{aligned}
$$

Diese Formel hat eine sehr anschauliche Bedeutung. Sie enthält nämlich einen $N$ proportionalen Anteil, zu welchem außer dem ersten Summanden auch noch ein Teil des zweiten gehört, und einen $N^{2}$ proportionalen Anteil, der bei großem $N$ den größten Teil des zweiten Summanden ausmacht. Haben wir nun lauter $\mathfrak{A}_{k}$ mit gleichem Betrag und gleicher Phase, so bleibt in (6) streng nur der $N^{2}$ proportionale Anteil stehen, wie sich leicht nachrechnen läßt, da dann alle Integrale nur über Konstanten zu erstrecken sind, und die beiden $N$-Glieder sich wegheben. Dies ist der Fall der Utberlagerung gleichphasiger Lichterregungen, wo sich die Amplituden addieren, und daher die Intensität das $N^{2}$-fache der Einzelintensität wird. Haben wir dagegen $\mathfrak{A}_{k}$ mit zwar gleichen Beträgen, aber gleichwahrscheinlich über alle Werte zwischen 0 und $2 \pi$ verteilten Phasen, so verschwinden die beiden Integrale mit dem $\cos \varphi$ und $\sin \varphi$ exakt, und es bleibt in (6) nur der $N$ proportionale Anteil übrig. Dies ist der Fall der Uberlagerung der Lichterregung mit statistisch verteilten Phasen, wo sich direkt die Intensitäten zur $N$-fachen Einzelintensität addieren. 
In jedem anderen Fall, wo eine Koppelung zwischen den Verteilungen der Beträge und der Phasen besteht, treten beide Glieder auf, so auch im Falle unseres Problems, den wir erhalten, wenn wir in (6) für $a$ den Betrag $\frac{R}{s} J_{0}$ und für $\varphi$ die Phase $(-k s)$ der komplexen Amplitude (5) einsetzen. Dann stellt (6) den gesuchten Erwartungswert der Lichtintensität in dem Raumpunkt $(x, y)$ dar.

Solange wir uns nun auf Punkten der Achse befinden, ist $y=0$ und $J_{0}=1$, so daß einfach $a=R / s$ wird, was eine im Vergleich $\mathrm{zu} \cos (k s)$ langsam veränderliche Funktion von $R$ ist, solange

bleibt.

$$
x \ll k R^{2}
$$

Andererseits macht $\cos (k s)$ im Integrationsbereich $\left(R_{2}-R_{1}\right)$ sehr viele Hin- und Hergänge. solange

ist.

$$
x \ll k\left(R_{2}^{2}-R_{1}^{2}\right)
$$

Die Bedingungen (7) und (8) folgen einfach aus einer Entwicklung von $s=\sqrt{x^{2}+R^{2}}(y=0)$ für $x \gg R$ und besagen beide ungefähr dasselbe. wenn unserer Voraussetzung gemäß $R_{1}$ von derselben Größenordnung wie $\left(R_{2}-R_{1}\right)$ ist. Dies ist einer der Gründe, warum man für die Berechnung mit $R_{1}$ nicht gegen Null gehen kann.

Bei Gültigkeit von (7) bzw. (8) mittelt sich der rasch schwankende $\cos (k s)$ in dem Integral in (6) fast völlig heraus, und die beiden Integrale des zweiten Summanden werden $\ll \bar{a}\left(R_{2}-R_{1}\right)$.

Etwas schwieriger ist die Sache bei Punkten außerhalb der Achse, wo zu a die Bessel-Funktion $J_{0}$ hinzutritt, die für großes Argument selbst eine rasch schwankende Funktion ist. Da ihre langsam mit $R$ veränderliche Periode jedoch von derjenigen von $\cos (k s)$ im allgemeinen verschieden ist - nur in einem Punkt des Integrationsintervalls können beide unter Umständen einander gleich werden -, haben wir über das Produkt von zwei nahezu sinusförmigen Funktionen mit verschiedener Periode zu integrieren, wobei sich ebenfalls fast alles weghebt, falls nur wieder genügend viele Hin- und Hergänge im Integrations. intervall liegen.

Wir erhalten also innerhalb der Bedingung hier für

$$
r \ll k R^{2}
$$

überall den Faktoren von $N^{2}$ in (6) sehr klein gegen denjenigen von $N$ und dürfen $\bar{S}^{2}$ nach der einfachen Integration über die Intensitäten:

$$
\overline{S^{2}} \approx \frac{N}{R_{2}-R_{1}} \int_{R_{1}}^{R_{2}} a^{2} d R
$$

errechnen, solange $N$ nicht $z$ u gro $\beta$ wird.

Das Interessante an der ganzen Überlegung ist nämlich, daß auch bei noch so kleinen Integralen im zweiten Summanden von (6) dieser für genügend großes $N$ überwiegt. Bei sehr großem $N$ bleibt schließlich von der Statistik nichts mehr übrig; die Lichtintensität errechnet sich genau so, als ob über alle infinitesimalen Ringzonen mit strenger Berücksichtigung ihrer Phase integriert würde, was nach dem Huygensschen Prinzip die ungestörte Ausbreitung der einfallenden ebenen Welle liefert. Die Ringplatte nähert sich demnach für extrem großes $N$, trotz statístischer Verteilung der Ringe, immer mehr einer klar durchsichtigen Platte (praktisch wegen der Unvollkommenheiten der Ringe wohl eher einer Mattscheibe).

Für Licht haben wir $k \approx 10^{5} \mathrm{~cm}^{-1}$; die Ungleichungen (7) und (8) sind also für $x \approx 1 \mathrm{~m}$ und für $R_{1}$ sowie $R_{2}-R_{1}$ in der Größenordnung von. Zentimetern noch sehr stark erfüllt. Eine Abschätzung der Integrale in (6) zeigt in diesem Fall, daß $N$ mindestens bis 10000 gehen darf, ehe das $N^{2}$ proportionale Glied gegen das erste merklich wird.

4. Das Beugungsfeld der Ringplatte Setzen wir $a=\frac{R}{s} J_{0}$ in (9) ein, so erhalten wir für den Erwartungswert der Lichtintensität:

$$
\overline{S^{2}}=C \int_{R_{1}}^{R_{2}} \frac{R^{2}}{R^{2}+r^{2}} J_{0}^{2}\left(\frac{k R y}{\sqrt{R^{2}+r^{2}}}\right) d R .
$$

Für kleines Argument von $J_{0}$ kann $J_{0}$ in eine Potenzreihe entwickelt werden, wobei der Integrand eine Summe von rationalen Ausdrücken in $R$ wird und gliedweise integriert werden kann. Für großes Argument von $J_{0}$ ersetzen wir $J_{0}$ durch seine asymptotische Darstellung.

Von der Potenzreihen-Entwicklung möge hier nur das Absolutglied behandelt werden, welches streng die Lichtintensität auf der Achse selbst darstellt. Die weiteren Glieder sind zwar unschwer berechenbar, bieten aber nicht viel Interesse, da man den Gesamtverlauf der Intensität 
aus dem Achs-Wert und der Näherung für großes Argument schon gut überblicken kann.

Für die Achse ist $J_{0}=1$ und daher

$$
\begin{aligned}
\overline{S^{2}} & =C \int_{R_{1}}^{R_{2}} \frac{R^{2} d R}{R^{2}+r^{2}} \\
& =C\left\{R_{2}-R_{1}-x \operatorname{arctg} \frac{x\left(R_{2}-R_{1}\right)}{R_{1} R_{2}+x^{2}}\right\} .
\end{aligned}
$$

Für $x \ll R_{1}$ wird dies

$$
\overline{S^{\prime}} \approx C\left(R_{2}-R_{1}\right)\left(1-x^{2} / R_{1} R_{2}\right),
$$

für $x \gg R_{2}$

$$
\overline{S^{2}} \approx C \frac{R_{2}^{3}-R_{1}^{3}}{3 x^{2}} \text {. }
$$

Der Verlauf entlang der Achse beginnt also mit einem Maximum bei $x=0$ unmittelbar hinter dem abgedeckten Zentrum der Ringscheibe und geht bei großem $x$ mit $1 / x^{2}$. Es ist in Abb. $2 \mathrm{~b}$ und $2 \mathrm{c}$ als dünne Linie eingezeichnet.

Nachher wird sich zeigen, daß die Intensität von der Achse weg äußerst steil abfällt. Wir haben also hier den „Brennstrahl“ der Ringscheibe vor uns. Stellen wir nun diesem im Abstand $x_{s}$ von der Ringscheibe eine kreisrunde, undurchsichtige Scheibe vom Radius $R_{s}\left(R_{s}<R_{1}\right)$ als ,Hinder. nis" in den Weg (Abb.2), so können wir zunächst zwei Punkte $x_{2}$ und $x_{1}$ auf der Achse konstruieren, indem wir Kegel durch den äußersten Ring $R_{2}$ bzw. den innersten Ring $R_{1}$ der Ringscheibe und den Umfang der Hindernisscheibe $R_{s}$ legen. Wir haben dadurch die Achse in 4 Abschnitte I bis IV eingeteilt.

Sehen wir nun von der nochmaligen Beugung am Rande der Hindernisscheibe ab, welche keine nennenswerte Intensitätsverschiebung liefert, so haben wir offenbar im Abschnitt I die ungestörte Intensität und im Abschnitt II die Intensität Null, da die dort liegenden Achs-Punkte von keinem der beugenden Ringe Licht erhalten können. Im Abschnitt III jedoch ist dies teilweise wieder der Fall. Für jeden Punkt $x$ dieses Abschnittes liefert ein entsprechender Kegel einen Radius $R_{x}$ zwischen $R_{1}$ und $R_{2}$ auf der Ringscheibe, und wir erhalten die Intensität in $x$, indem wir das Integral in (11) statt von $R_{1}$ nur von $R_{x}$ bis $R_{2}$ erstrecken.
Im Punkt $x_{1}$ ist ersichtlich die volle Intensität wieder erreicht, die von da ab im Abschnitt IV erhalten bleibt. Abb. 2 b zeigt den Verlauf der Intensität entlang der Achse; der „Strahl“ erscheint bei $x_{2}$ wieder und erreicht bei $x_{1}$ seine ungestörte Intensität.

Ganz entsprechend liegen die Verhältnisse, wenn wir statt des Hindernisses eine Lochblende vom Radius $R_{s}$ bei $x_{s}$ anbringen (Abb. $2 \mathrm{c}$ ). Jetzt geht der „Strahl“ zwar ungestört bis $x_{2}$, nimmt aber von da an gegenüber seiner ungestörten Intensität ab und erlischt bei $x_{1}$ völlig. Die Ubergangsfunktionen im Gebiet III sind mit Hilfe des Integrals (11) auszurechnen.

Ist $R_{s}>R_{1}$, so existiert der Punkt $x_{1}$ nicht. Der ,Strahl“" erreicht dann hinter dem Hindernis nirgends wieder seine ungestörte Stärke bzw. erlischt hinter der Lochblende nie völlig. a)

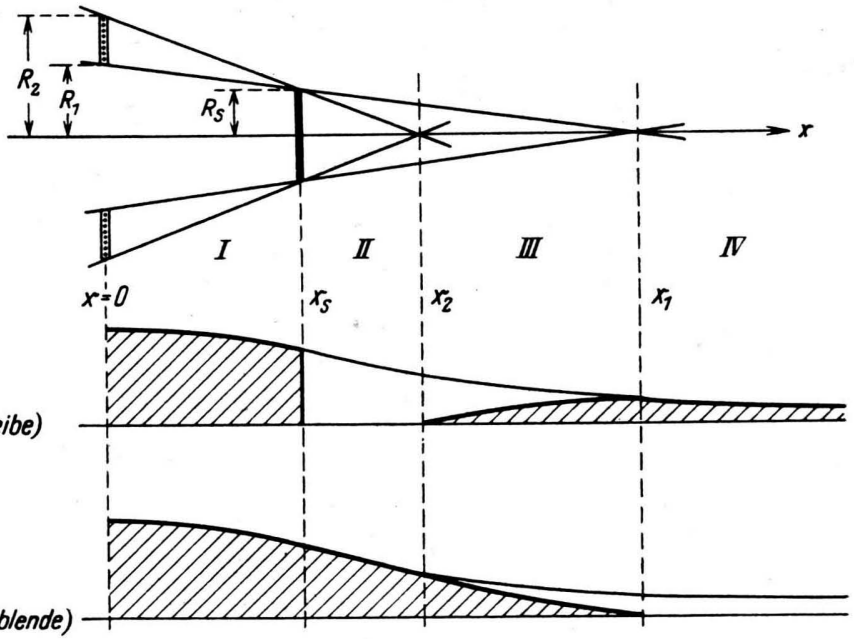

Abb. 2. Verhalten des „Brennstrahls“ nach einem Hindernis und nach einer Lochblende.

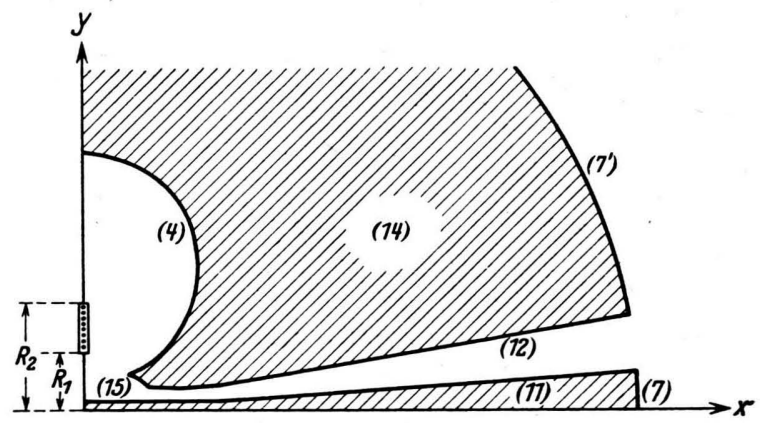

Abb. 3. Die von den Gln. (11) und (14) beherrschten Gebiete (nicht maßstäblich). 
Außerhalb der Achse ersetzen wir nun, falls

$$
\frac{k R y}{s} \gg 1
$$

ist (dies trifft wegen $k R \approx$ einige $10^{5}$ schon für Winkel $y / x$ von weniger als einer Bogenminute zu), die Bessel-Funktion durch ihre asymptotische Darstellung:

$$
J_{0}\left(\frac{k R y}{s}\right) \approx \sqrt{\frac{2 s}{\pi k R y}} \cos \left(\frac{k R y}{s}-\frac{\pi}{4}\right) .
$$

Damit wird (10):

$$
\overline{S^{2}}=C \frac{2}{\pi k y} \int_{R_{1}}^{R_{2}} \frac{R}{s} \cos ^{2}\left(\frac{k R y}{s}-\frac{\pi}{4}\right) d R .
$$

Liegen im Integrationsintervall genügend viele Perioden des cos, so darf der $\cos ^{2}$ durch seinen Mittelwert 1/2 ersetzt werden, und es wird wegen $R d R=s d s$

$$
\overline{S^{2}}=\frac{C}{\pi k y} \int_{s_{1}}^{s_{2}} d s=\frac{C\left(s_{2}-s_{1}\right)}{\pi k y} .
$$

Die Bedingung dafür, daß sehr viele Perioden des cos im Integrationsintervall liegen, ist, da die Periodenlänge die Größenordnung

$$
\begin{gathered}
1: \frac{d}{d R}\left(\frac{k R y}{s}\right)=\frac{s^{3}}{k r^{2} y} \text { hat, } \\
\frac{s^{3}}{k r^{2} y} \ll R_{2}-R_{1} .
\end{gathered}
$$

Dies ist für großes $r$, wo $s \approx r$ ist, wegen (12) von selbst erfüllt, gibt jedoch bei kleinem $r$, wo $s \approx R$ wird, eine zusätzliche Einschränkung, wodurch ein kleines Gebiet um den Mittelpunkt der Ringscheibe herum von der Anwendbarkeit der Formel (14) ausgeschlossen wird.

Abb. 3 zeigt schematisch (die $\ll$ und $\gg$ sind hier der Deutlichkeit halber sehr großzügig ausgelegt) die Begrenzung des Anwendungsgebietes der Gl. (14), wobei an den einzelnen Grenzen jeweils die begrenzende Bedingung angeschrieben ist. Unmittelbar um die Achse herum liegt dann noch das Gebiet der Gl. (11) mit ihrer Erweiterung durch die Potenzreihenentwicklung von $J_{0}$. Das zwischen beiden verbleibende Gebiet enthält den Übergang zwischen (11) und (14).

Für konstantes $x$ liefern nun (11) und (14) einen ,Querschnitt“" des Brennstrahls. (11) gibt das auf der Achse liegende Maximum, (14) den Abfall nach außen. Für großes $r$ können die $s$ in (14) entwickelt werden, und wir erhalten

$$
\overline{S^{2}} \approx \frac{C\left(R_{2}^{2}-R_{1}^{2}\right)}{2 \pi k y \sqrt{x^{2}+y^{2}}} .
$$

Die Intensität nimmt bei großem, konstantem $x$ also zunächst wie $1 / y$, sehr weit draußen wie $1 / y^{2}$ ab.

Wir können eine „Breite“ $2 y_{\mathrm{b}}$ des Brennstrahls definieren, wenn wir für festes $x$ nach Abb. 4 den Schnittpunkt von (11) und (14) bestimmen. Für $y_{\mathrm{b}}$ kommt durch Gleichsetzen des $\bar{S}^{2}$ nach (11) und (14) eine transzendente Gleichung, welche sich für $x \ll R_{1}$ und $x \gg R_{2}$ näherungsweise lösen läßt. Wir erhalten für die halbe Breite:

$$
\text { für } x \ll R_{1}: y_{\mathrm{b}} \approx \frac{1}{\pi k}\left(1+\frac{x^{2}}{2 R_{1} R_{2}}\right),
$$

für $\mathrm{x} \gg R_{2}: y_{\mathrm{b}} \approx \frac{3\left(R_{1}+R_{2}\right) x}{2 \pi k\left(R_{1}^{2}+R_{1} R_{2}+R_{2}^{2}\right)} \approx \frac{x}{\pi k R}$.

Da $1 / \pi k=\lambda / 2 \pi^{2}$ ist, beträgt die ..Breite" des Strahls für $x=0$ nur Bruchteile einer Lichtwellenlänge und wächst für große $x$ proportional zu $x$ an mit einem äußerst kleinen Öffnungswinkel von noch nicht einer Bogensekunde bei einem mittleren Ringradius $\bar{R}$ von einigen $\mathrm{cm}$. Bei praktischen Versuchen wird daher die durch die Gln. (16a) und (16b) gegebene Schärfe des Brennstrahls nicht im entferntesten erreicht, einmal wegen der nicht exakten Punktförmigkeit der Lichtquelle, und zum andern, weil die einzelnen Ringe der Platte nicht exakt konzentrisch sind.

Man darf auch angesichts der Gl. (16) nicht vergessen, daß ein großer Teil des "Strahls" außerhalb der nach Abb. 4 definierten „Breite“

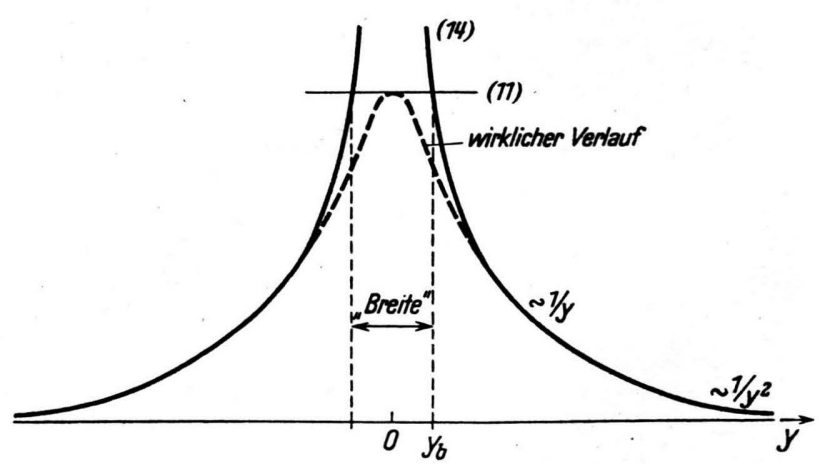

Abb. 4. „Querschnitt“ des „Brennstrahls“ (nicht maßstäblich). 
läuft. Für kleines $y$ ist zwar der Abfall mit $1 / y$ recht steil, im ganzen bedeutet er aber doch einen schwachen Abfall nach außen, der erst für sehr große $y$ in $1 / y^{2}$ übergeht.

Rechnen wir den Energiefluß durch eine Kugelfläche um den Scheibenmittelpunkt aus - für großes $r$ steht der Energiestrom überall näherungsweise senkrecht auf dieser Fläche, so daß einfach über die Intensität zu integrieren ist so erhalten wir mit (14b) außerhalb des durch die Breite definierten Kegels:

$$
\begin{aligned}
E_{1} & =\int_{y_{\mathrm{b}} / x}^{\pi / 2} \overline{S^{2}} 2 \pi \sin \varphi r^{2} d \varphi, \quad(\varphi=\Varangle(r, x)) \\
& =\frac{C\left(R_{2}^{2}-R_{1}^{2}\right)}{k}\left(\frac{\pi}{2}-\frac{y_{\mathrm{b}}}{x}\right) \approx \frac{\pi}{2} \frac{C\left(R_{2}^{2}-R_{1}^{2}\right)}{k} .
\end{aligned}
$$

Dies ist, wie es sein muß, unabhängig vom Radius $r$ der Kugelfläche. Innerhalb des $y_{\lrcorner}$-Kegels dagegen erhalten wir mit (11 b), wenn wir wieder statt der wirklichen die eckige Intensitätsverteilung in Abb. 4 zugrundelegen,

$$
E_{2} \approx C \frac{R_{2}^{3}-R_{1}^{3}}{3 x^{2}} \int_{0}^{y_{\mathrm{b}} / x} 2 \pi x^{2} \varphi d \varphi \approx \frac{\pi C\left(R_{2}^{3}-R_{1}^{3}\right)}{3} \frac{y_{\mathrm{b}}}{x^{2}} .
$$

Es wird also (mit 16b):

$$
E_{1} / E_{2} \approx k \bar{R}\left(y_{\mathrm{b}} / x\right)^{2} \approx 1 / \pi^{2} k \bar{R},
$$

d. h. nur ein Bruchteil von weniger als $10^{-6}$ der ganzen Energie fließt durch den $y_{0}$-Kegel. Dies liegt natürlich daran, daß die Flächen der Ringzonen bei der Integration nach außen zunächst mit $y$ zunehmen, während die Intensität nur mit $1 / y$ abnimmt. Der „Brennstrahl“ ist eben durchaus nicht so scharf nach außen begrenzt, wie es dem Auge, welches Kontraste übertrieben empfindet, erscheint.

\title{
Die Bildung reflexvermindernder Oberflächenschichten auf Gląs durch Lösungen mit $p_{\mathrm{H}}$-Werten nahe 7
}

\author{
Von Hubert Schröder \\ Aus dem Laboratorium der Optischen Werke C. A. Steinheil Söhne G.m.b.H. München \\ (Z. Naturforschg. 4a, 515-521 [1949]; eingegangen am 6. April 1949) \\ Herrn Prof. Dr. W. Gerlach zum 60. Geburtstag gewidmet.
}

\begin{abstract}
Wäßrige Lösungen mit $p_{\mathrm{H}}$-Werten nahe dem Neutralpunkt können auf polierten Oberflächen der meisten Glasarten reflexvermindernde Schichten erzeugen, deren Bildung durch partiellen, an Inhomogenitätsstellen ansetzenden Angriff auf die Kieselsäure begründet wird. Die Zahl der erzeugten Poren wird zu $5-10 \cdot 10^{11} / \mathrm{cm}^{2}$ Glasoberfläche ermittelt. Die Untersuchung der Kinetik der Schichtbildung ergibt bei geeigneten Lösungen verminderte Aktivierungsenergien für den partiellen Angriff gegenüber dem totalen Glasabbau. Das Geschwindigkeitsverhältnis der beiden nebeneinander herlaufenden Korrosionsvorgänge ändert sich mit zunehmender Dicke der gebildeten Schicht, was mit der Wirkung der auf diese Weise dickenmäßig bestimmbaren „Polierschicht“ in $\mathrm{Zu}$ sammenhang gebracht wird.
\end{abstract}

$I^{\prime \prime}$ $n$ früheren Mitteilungen ${ }^{\mathbf{1 , 2}}$ wurde über Eigenschaften von reflexvermindernden Oberflächenschichten berichtet, welche man durch chemische Einwirkung, insbesondere von Säuren oder sauren Salzen, auf optischen Gläsern erzeugen kann. Neben der Untersuchung der fertigen Schichten wurden dabei auch die Auslaugungsvorgänge selbst beobachtet, die sich bei der damals an-

1 H. S ch rö d er, Glastechn. Ber. 20, 161 [1942].

${ }^{2}$ H. S c h r öd e r, Z. techn. Physik 22, 38 [1941] u. 23, 196 [1942]. gewandten Behandlung in erster Linie auf säurelösliche Bestandteile der Gläser erstreckten, während das Kieselsäuregerüst wegen seiner viel geringeren Lösungsgeschwindigkeit, wenigstens primär, unverletzt blieb. Ein solches Verhalten zeigen die meisten blei-, barium- oder zinkhaltigen Gläser, wohingegen die einfachen Kalk-NatronSilikatgläser bei der Säurebehandlung optisch nahezu unverändert bleiben. Die erreichte Porosität der Oberfläche wird in den betrachteten Fällen einfach durch den Gehalt an löslichen Komponen- 